\title{
Estudio Radiográfico y Electromiográfico de los Músculos Masetero y Temporal Anterior en Individuos con Maloclusión Tipo II, 1 de Angle y Controles
}

\author{
Radiographic and Electromiographic Study of Masseter and Temporal \\ Anterior Muscles in Subjects with Angle II, I Malloclusion and Controls
}

"Albornoz, M.; ** Ogalde, A. \& ${ }^{* * *}$ Aguirre, M.

\begin{abstract}
ALbORNOZ, M.; OGALDE, A. \& AGUIRRE, M. Estudio radiográfico y electromiográfico de los músculos masetero y temporal anterior en individuos con maloclusión Tipo II, 1 de Angle y controles. Int. J. Morphol., 27(3):861-866, 2009.

RESUMEN: El objetivo de este estudio fue comparar la actividad electromiográfica (EMG) de los músculos Masetero (Ms) y Temporal anterior (Ta) de sujetos portadores de maloclusión Angle II, 1 y controles. Se seleccionaron 19 sujetos estudiantes universitarios con características antropométricas similares (edad, peso, talla e IMC). Clínica y radiográficamente se dividieron en dos grupos: Grupo Control (GC) individuos portadores de neutroclusión y Grupo Disto (GD) con individuos portadores de maloclusión Tipo II, 1 de Angle. Se les realizó una electromiografía de superficie (EMG) de acuerdo a la técnica de Ferrario et al. (1993) y se analizaron los datos a partir de Root Mean Square (RMS). A partir de este registro se analizaron la amplitud media de voltaje (mV), la relación Ms/Ta y la distribución de las clases de voltaje, determinadas por los potenciales de acción de unidades motoras (PAUM). De la telerradiografía se analizaron los siguientes datos: ANB, SNA, SNB según Steiner (1992), relación molar, ángulo alfa de inclinación del Ms y brazo de palanca de acuerdo al modelo de Throkcmorton et al. (1980). Se utilizó Test "t" Student para evaluar significancia estadística $(p<0,05)$ entre los grupos. La amplitud de voltaje fue mayor en el GD para todos los músculos estudiados ( $<0,05)$. La relación Ms/Ta fue mayor en el GC. El ángulo alfa fue mayor en GD, también el brazo de palanca. La distribución de PAUM fue mayor en GC (79,0\%), que en GD $(57,8 \%)(\mathrm{p}<0,05)$. GD presenta mayor amplitud de voltaje que GC, probablemente por presentar menos aferencias inhibitorias periodontales. También GD presenta una menor variedad de PAUM lo que implica que tiene menos reserva funcional que GC.
\end{abstract}

PALABRAS CLAVE: Electromiografía; Músculos masetero y temporal anterior; Maloclusión Tipo II, 1 de Angle.

\section{INTRODUCCIÓN}

Las maloclusiones presentan un porcentaje importante de prevalencia en la población (Hebling et al., 2008; Traebert \& Peres, 2005; Grando et al., 2008; Sidlauskas \& Lopatiene, 2009). Algunos autores informan de una prevalencia de hasta un $88 \%$, hecho que constituye a esta anomalía en un problema de salud pública, ya que el sistema estomatognático compromete la vía aérea superior y al sistema digestivo en las funciones de masticación, salivación y deglución. Las maloclusiones son de origen multicausal, genético, mediambiental o ambas, determinando un desequilibrio entre las fuerzas que gobiernan al sistema estomatognático. Angle (1907) definió las maloclusiones en base a la relación entre los arcos dentarios en I, II y III
(Sabashi et al., 2009). Este diagnóstico se puede completar con un examen radiográfico que determina a partir de una cefalometría la relación entre las bases óseas, base de cráneo, maxilar y mandíbula.

El sistema estomatognático consta, estructuralmente, de bases óseas, articulación temporomandibular y componentes neuromusculares que interactúan entre sí. La musculatura permite determinar la postura y los movimientos mandibulares. La actividad muscular se evalúa por electromiografía (EMG) de superficie, a partir de la cual se pueden determinar los potenciales de acción de unidad motora (PAUM) (Angle; Silvestre, 2002; Ogalde \& Cardenas, 2000).

\footnotetext{
* Magister en Ciencias Mención Fisiología. Departamento de Fisiología, Facultad de Ciencias Biológicas. Universidad de Concepción. Profesora Asistente. Dirección de Ciencias Biomédicas. Universidad Autónoma de Chile - Talca.

** Profesor Asistente. Departamento de Fisiología, Facultad de Ciencias Biológicas. Universidad de Concepción.

${ }^{* * * *}$ Profesor Asociado, Vicedecano Facultad de Odontología. Universidad de Concepción.
} 
Este estudio evaluará la actividad muscular de músculos elevadores mandibulares en sujetos portadores de malocusión Angle II, 1 y controles, para observar el impacto de la arquitectura craneofacial en la función muscular.

\section{MATERIAL Y MÉTODO}

Este estudio comprendió un grupo de 19 individuos, todos estudiantes de pregrado, segundo nivel de las diferentes carreras del área de la salud de la Universidad de Concepción. Todos los pacientes fueron previamente informados sobre el estudio al que serían sometidos y se integraron sólo aquellos que dejaron expreso su consentimiento.

Se formaron dos grupos, uno de individuos con neutro oclusión (GC) y otro con individuos con disto oclusión Tipo II, 1 de Angle. La formación de los grupos GC y GD de estudio se determinó en base al siguiente protocolo:

Examen clínico del paciente. Mediante este examen se realizó una inspección visual de la mordida para ver las relaciones entre los arcos dentarios.

Examen radiográfico. Mediante una telerradiografía en norma lateral se determinaron las relaciones entre las bases óseas basadas en los ángulos SNA, SNB y ANB de Steiner. Ambos grupos cumplieron las siguientes condiciones: presentar características antropológicas similares (edad, peso, ta1la), presentar fórmula dentaria completa (excepto terceros molares), ausencia clínica de desórdenes mandibulares como chasquido, crepitación de la articulación temporomandibular, dolor a la palpación de músculos masticatorios, no deben presentar alteraciones musculares, no deben estar sometidos a tratamiento ortodóntico, restauraciones dentales en buen estado, ausencia de patologías periodontales, no deben estar sometidos a tratamiento farmacológico.

Registro Cefalométrico. El análisis cefalométrico fue hecho usando una telerradiografía de norma lateral en Posición de Máxima Intercuspidación (PMI) en todos los individuos. Para ello se uso un aparato de radiografías Crane $\mathrm{X}$ Tome y un equipo revelador de placas radiográficas AGFA Curix 60. Mediante la utilización de la telerradiografía lateral se logró determinar el tipo de oclusión del paciente en base a: SNA, SNB, ANB, Gonion, relación molar, ángulo alfa, inclinación de la fibras del Ms (Throkcmorton et al., 1980). El ángulo a está formado por el plano mandibular (Go-Me) y la fuerza vector de las fibras del masetero en el área exterior medial. Este vector es la línea que conecta el Go con la articulación entre el hueso frontal y el hueso cigomático.
Electromiografía (EMG). La EMG en norma bipolar se realizó utilizando electrodos de superficie según las técnicas de Ferrario et al.; Haraldson et al. (1985); Deguchi et al. (1994). Se colocó al paciente sentado, espalda recta, plano de Francfort paralelo al suelo, dentro de una jaula de Faraday conectada a tierra. Los electrodos de superficie utilizados fueron de $10 \mathrm{~mm}$ $3 \mathrm{M}$ código 2222. El electrodo de control se ubicó en la parte más prominente del vientre del músculo Ms y Ta en contracción isométrica. El segundo electrodo se ubicó a $15 \mathrm{~mm}$. del electrodo de control siguiendo la dirección de las fibras musculares. Los electrodos de tierra para cada músculo se ubicaron en el mentón. El registro EMG fue llevado, desde los electrodos, mediante cables fusionados Biopac cod. (SS1L electrode SN 7094) a una tarjeta convertidora análogo-digital (biopac MP30). Todos los impulsos eléctricos convertidos en guarismos se almacenaron en un computador con la ayuda del software V 3.66. La captura de señales del registro EMG fue de $5000 \mathrm{~Hz}$. Se realizaron tres registros EMG en PMI, cada uno de los cuales duró 6 segundos. De los tres registros se eligió para el análisis el que presentó un patrón de interferencia estable y de mayor amplitud de voltaje. Del registro elegido se seleccionó 1 segundo.

Con los valores obtenidos se calculó el RMS para comparar los promedios de amplitud de voltaje entre los grupos, además se ordenaron según su frecuencia y graficaron en un eje de coordenadas que tenía las clases de voltajes (intervalos de $50 \mu$ Volt), obteniéndose un histograma de distribución de frecuencias v/s clases de voltaje. Las clases de voltaje correspondes a PAUM. Se promediaron todos los valores que conforman la onda EMG de cada registro EMG, esto se realizó con el fin de obtener un número absoluto que represente el promedio de mVolts generados por el PAUM respectivo. De esta forma se pueden obtener datos comparables entre los distintos registros electromiográficos.

Las pruebas de significancia estadística se realizaron utilizando la prueba Test "t" de Student con un $\mathrm{p}<0.05$.

\section{RESULTADOS}

Los resultados se expresan desde la Tabla I a la Tabla VII.

\section{DISCUSIÓN}

Según las evaluaciones clínicas y clasificaciones antropométricas realizadas a los sujetos elegidos, obtuvimos dos grupos homogéneos que no mostraban diferencias 
ALbORNOZ, M.; OGALDE, A. \& AGUIRRE, M. Estudio radiográfico y electromiográfico de los músculos masetero y temporal anterior en individuos con maloclusión Tipo II, 1 de Angle y controles. Int. J. Morphol., 27(3):861-866, 2009.

Tabla I. Media y desviación estándar (DS) de las características antropométricas de los GC y GD.

\begin{tabular}{lcccccc}
\hline & $\mathbf{n}$ & Edad (años) & Peso $(\mathbf{K g})$ & Talla $(\mathbf{c m})$ & IMC & Género \\
\hline GC & 8 & $19 \pm 0$ & $71.8 \pm 9.44$ & $173.8 \pm 6.24$ & $23.61 \pm 3.12$ & $3 \mathrm{M}-5 \mathrm{~F}$ \\
GD & 11 & $19 \pm 1.43$ & $59.67 \pm 3.22$ & $164.57 \pm 3.35$ & $22.24 \pm 1.47$ & $4 \mathrm{M}-7 \mathrm{~F}$ \\
\hline
\end{tabular}

Tabla II. Valores normales. Media. Desviación estándar (DS) de ángulos y factores cefalométricos en los GC Y GD.

\begin{tabular}{lrrc}
\hline & \multicolumn{1}{c}{ GC } & \multicolumn{1}{c}{ GD } & Valores Normales \\
\hline SNA & $81,25 \pm 3,75$ & $81,44 \pm 2,06$ & $82 \pm 2$ \\
SNB & $79,00 \pm 2.50$ & $75,88 \pm 2.13$ & $80 \pm 2$ \\
ANB & $2,25 \pm 0,38$ & $5,19 \pm 1,41$ & 2 \\
Gonion & $118,50 \pm 2,00$ & $124,75 \pm 5,56$ & $128 \pm 7$ \\
Relación molar & $3,5 \pm 0,20$ & $-3,3 \pm 1,63$ & $4 \mathrm{~mm}$ \\
\hline
\end{tabular}

Tabla III. Angulo alfa y brazo de palanca del Ms en los GC y GD.

\begin{tabular}{cccc}
\hline & $\mathbf{n}$ & $\begin{array}{c}\text { Ángulo alfa } \\
\text { (grados) }\end{array}$ & $\begin{array}{c}\text { Brazo de palanca Ms } \\
(\mathbf{m m})\end{array}$ \\
\hline GC & 8 & 83.6 & 3.7 \\
GD & 11 & 86.7 & 3.2 \\
\hline
\end{tabular}

Tabla V. Valores de la relación Ms/Ta en GC y GD.

\begin{tabular}{cccc}
\hline & $\mathbf{n}$ & Lado Derecho & Lado Izquierdo \\
\hline GC & 8 & 1.17 & 1.20 \\
GD & 11 & 0.85 & 0.69 \\
\hline
\end{tabular}

Tabla VII. Rango de clases de voltaje PAUM entre 50 - 4000 mvolt y 80 clases, en GC y GD. Promedio de Porcentajes de rango de voltajes. $\mathrm{P}<0,05$ según Test " $t$ Student".

\begin{tabular}{ccc}
\hline & n & \% de rango de voltajes \\
\hline GC & 8 & 79.01 \\
GD & 11 & 57.8 \\
\hline
\end{tabular}

significativas en cuanto a edad, peso, talla, IMC (GC similar a GD), por cuya razón nos permitieron un estudio objetivo de las variables a analizar (Tabla I). Los que nos permite descartar el efecto de la variable masa en la actividad EMG.

Los grupos GC y GD se ordenaron de acuerdo a los resultados obtenidos del análisis cefalométrico. GC se acerca bastante a los valores de referencia en los ángulos SNA y SNB para individuos con neutroclusión. Los ángulos SNA y SNB describen la relación entre bases óseas, es decir, SNA informa de la relación de posición del maxilar con respecto a la base de cráneo y SNB informa de la posición de la mandíbula con respecto a la base de cráneo. ANB representa la
Tabla IV.Valores de RMS en Ms y Ta (der-iz) en GC y GD. Estadís ticamente significativa con un $\mathrm{P}<0,05$ seg ún Test "t Student".

\begin{tabular}{lccccc}
\hline & $\mathbf{n}$ & MsDer & TaDer & MsIz & TaIz \\
\hline GC & 8 & 63.63 & 54.38 & 54.25 & 45.25 \\
GD & 11 & 73.00 & 86.00 & 65.82 & 96.00 \\
\hline
\end{tabular}

Tabla VI. Valores promedios de frecuencia máxima en Ms y Ta (der-iz) en GC y GD. Estadísticamente significativa con un $\mathrm{P}<$ 0,05 según Test "t Student".

\begin{tabular}{lrcccc}
\hline & n & MsDer & TaDer & MsIz & TaIz \\
\hline GC & 8 & 972 & 1291 & 950 & 1157 \\
GD & 11 & 513 & 527 & 544 & 425 \\
\hline
\end{tabular}

relación de posición entre los maxilares. GD presenta el SNA muy cercano a la normalidad y el SNB con una desviación de aproximadamente 4 grados de la norma lo que corrobora la condición de distoclusión verdadera (Tabla II). Esto se reafirma con los valores de ANB que en GC es muy cercano a la norma y en GD se desvía en 3 grados. También la relación molar, que es determinante de la relación de los arcos dentarios, es normal en GC, cercana a $4 \mathrm{~mm}$. En GD la relación molar promedio es típica de oclusión distal acercándose a los $-3,3 \mathrm{~mm}$., considerándose desde el cero una distoclusión. Por lo tanto, GD presenta la condición de distoclusión muy depurada tanto en la relaciones de las bases óseas como en la relación de los arcos dentarios. Otro índice cefalométrico que es típico en la arquitectura craneofacial distal mandibular, es el ángulo Goniaco (Go) mayor que la norma. En este caso GD presenta un Go mayor que la norma en 4 grados, lo que puede incidir en la baja de la ventaja mecánica según el modelo de Throckmorton et al. Sin embargo, el ángulo del Ms es mayor significativamente en GD con respecto a GC lo que implica que el componente 
vertical de la descomposición vectorial es mayor, posibilitando la generación de una mayor fuerza.

Esto también concuerda en que el brazo de palanca del Ms es más corto en GD (Tabla III). En nuestros estudios vemos que en el sistema mandibular con menor ventaja mecánica requeriría una musculatura relativamente mayor (más masa) para producir la fuerza que un sistema más eficaz pudiera generar con músculos más pequeños. Si el tamaño de los músculos permanece constante y la ventaja mecánica varía al modificar la longitud del brazo de carga, la fuerza masticatoria resultante fluctúa de acuerdo con las alteraciones en la ventaja mecánica. Esto explica en parte porque es posible aplicar más fuerza con los molares que con los incisivos (Throckmorton et al.).

Al analizar la amplitud de voltajes (Tabla IV) observamos que el GD presenta una amplitud de voltaje mayor que el GC con una diferencia estadísticamente significativa en todos los músculos estudiados. Esto nos indica que el GD está realizando una mayor tensión, ya que, la amplitud de voltaje registrada en una EMG, en contracción isométrica, es proporcional a la fuerza que desarrolla el músculo (Robertson et al., 2004). Según Miralles et al. (1991) no existen diferencias significativas en el promedio de amplitud de voltaje en las diferentes arquitecturas craneofaciales durante contracción máxima voluntaria en PMI. Si encuentra diferencias entre las arquitecturas craneofaciales en movimientos funcionales de trabajo submáximo presentando mayor amplitud promedio de voltaje los individuos de maloclusión clase III. Moreno et al. (2008) nos informan que los individuos con maloclusión Angle II, 1 presentan una amplitud del voltaje mas alta que el control para el Ta. Tomando en cuenta que la longitud del músculo es crítica en la generación de la tensiónactiva y tomando en cuenta los diversos vectores que determinan el equilibrio de postura mandibular, según Throckmorton et al., necesariamente deberíamos encontrar diferencias de respuesta eléctrica muscular en los distintos tipos de arquitectura facial, ya que habría variación en la longitud de los músculos y en los vectores que determinan las bases óseas. En este estudio el GD presenta el ángulo alfa del Ms cercano a los $90^{\circ}$, lo que determina a partir de la descomposición vectorial un componente vertical mayor que el GC, apoyando la observación de mayor amplitud de voltaje en GD. Ambos grupos presentan una variación, aunque discreta, de la longitud del Ms hecho que puede generar una tensión activa diferente. Aunque existiría en cada sujeto una longitud muscular optima de mayor eficiencia donde el músculo desarrolla la mayor fuerza muscular (Robertson et al., 2004).

Ante la presencia de un tipo de arquitectura diferente a la neutroclusión podemos esperar que el sistema neuro- muscular-esqueletal presente variaciones compensatorias para conservar la eficiencia masticatoria o la integridad estructural y funcional del sistema (Ferrario et al.).

Un punto controversial que se nos presentó al contrastar la literatura revisada con nuestros resultados fue el hecho que ésta informa que a menor número de contactos oclusales los sujetos de estudio presentan menor amplitud de voltaje (Ferrario et al.). Cabe destacar que dichos estudios fueron realizados entre individuos normales, es decir, cercanos a la neutroclusión. En nuestro caso los individuos de GD presentan una inoclusión anterior (Angle), lo que nos haría suponer que deberían presentar una menor amplitud de voltaje al tener menos puntos de contacto oclusal. Sin embargo, el GD presenta mayor amplitud de voltaje que el GC a pesar de tener menos puntos de contacto. Esto podría deberse a que presentan menos aferencias periodontales inhibitorias. Estas aferencias tendrían una función protectora del sistema, y al no tenerlas GD realiza mas fuerza relativa que GC. La literatura informa que en normalidad morfofuncional la propiocepción del sistema estomato-gnático es muy efectiva para limitar la contracción de los músculos elevadores mandibulares, con el fin de proteger las estructuras de las articulaciones temporomandibulares y las articulaciones dentarias especialmente en la posición más retruída del cóndilo y en PMI (Turkawski \& Van Eijden, 2000). De esta manera el GD estaría realizando de esta forma su función, pero trabajando de manera distinta con respecto al GC.

Otro parámetro que nos indica una forma distinta de trabajo en ambos grupos es la relación Ms/Ta; GD presenta mayor protagonismo del Ta; GC presenta mayor protagonismo del Ms (Tabla V). Pancherz (1980a) informa que, en niños neutroclusión, esta relación, se aproxima a la unidad y en la medida que se llega a la adultez la actividad eléctrica del Ms es mayor. Otro estudio del mismo autor (Pancherz, 1980b), pero en este caso en individuos disto Angle II, 1 informa que presentan una actividad EMG menor relativa del Ms con respecto al temporal, lo que estaría de acuerdo con nuestros registros. No así en la amplitud porque los controles de dicho estudio presentan mayor amplitud de voltaje.

Una contribución fundamental de nuestro estudio es el análisis de los PAUM, distribuidos en clases de voltaje (Ogalde \& Cardenas). El histograma entrega una rica información que nos puede ayudar a entender la adaptación a la función que hacen los individuos disto II, 1. La distribución en 80 clases de voltaje para la actividad eléctrica de todos los músculos estudiados (Ms y Ta), muestra una menor variedad de unidades motoras. En GD equivale a un $57.8 \%$, en GC, prácticamente se acerca al 79.01\% (Tabla VII). El GD presentó altas frecuencias de PAUM de bajo voltaje con 
respecto a GC (Tabla VI). De esta forma, podemos decir que GD consigue un mayor voltaje a expensas de un mayor reclutamiento de UM de menor voltaje. GC presenta una menor amplitud de voltaje pero tiene una mayor variedad de PAUM con distintos voltajes, destacando UM con mayor voltaje con respecto a GD. Es interesante observar que GC poseería una reserva funcional de UM que no tiene GD. Por lo tanto, GD estaría trabajando más para compensar las desviaciones que implica la diferente arquitectura craneofacial.

\section{CONCLUSIÓN}

Los resultados de nuestros estudios han comprobado nuestras teorías, puesto que en el primer parámetro analizado "Promedio de Voltajes (RMS)" se ha encontrado una significativa diferencia en los grupos. Ambos (GC y GD) re- gistran actividad eléctrica con distinta amplitud. De acuerdo a los resultados, la mordida del grupo GD es de mayor amplitud de voltaje que la del GC para ejercer su función, probablemente por presentar menos aferencias inhibitorias periodontales. También se evidenció que el GD presenta una menor variedad de PAUM lo que implica que tiene menos reserva funcional que GC, concluyendo así que los individuos del GD se adaptan a la función trabajando en forma distinta, optimizando el uso de sus UM.

\section{AGRADECIMIENTOS}

Los autores expresan sus agradecimientos al Departamento de Fisiología de la Facultad de Ciencias Biológicas de la Universidad de Concepción y a la Sección de Rayos X de la Facultad de Odontología de la Universidad de Concepción, por su colaboración en la realización de este trabajo de investigación.

ALBORNOZ, M.; OGALDE, A. \& AGUIRRE, M. Radiographic and electromiographic study of masseter and temporal anterior muscles in subjects with Angle II, I malloclusion and controls. Int. J. Morphol., 27(3):861-866, 2009.

SUMMARY: The of this study is compare the electromiographic activity (EMG) of the Masseter (Ms) and Temporal Anterior (Ta) muscles in subjects with malocclusion Angle II, I and controls. This study involving 19 young adults with similar anthropometric characteristics (Age, weight, hight, BMI). We evaluate clinical and radiographicaly this subject and divided it in to groups: Control Group CG with neutrocclusion and Distal Group (DG) with Angle II, I malocclusion. The both groups was submit a surface EMG according to Ferrario et al. (1993). We used the root mean square (RMS) math operation to calculate the mean voltage amplitude for each muscle. Furthermore we calculate the ratio $\mathrm{Ms} / \mathrm{Ta}$ and the distribution of voltage class determinate for motor units actions potentials (MUAP). The Rx examination included SNA, SNB and ANB angles according to Steiner (1992), alfa angle and lever arm of Ms according the Throckmorton et al. (1980) mandibular model. The Stundent " $t$ " Test was used to determine whether there were significant differences $(\mathrm{p}<0.05)$ between CG and DG parameters. The voltage amplitude was more higher in DG than CG. The ratio Ms/Ta showed protagonism of Ms in CG. The alfa angle and lever arm of Ms were more greater in DG than CG. DG presents a poor number of voltage class (57.8\%) respect to CG $(79.0 \%)$. DG presents a greater voltage amplitude in relation to CG, probably because the feedback inhibitory periodontal tissue plays an important role. Moreover DG presents a low voltage classes distribution of MUAP that suggest that have minus functional reserve.

KEY WORDS: Electromiography; Masseter and Temporal anterior muscles; Malocclusion AngleII, I.

\section{REFERENCIAS BIBLIOGRÁFICAS}

Angle, E. Treatment of malocclusion of the teeth Angles System. Philadelphia, S.S. White Dental Manufacturing Company, 1907. pp. 28-59.

Cardenas, H. \& Ogalde, H. Relationship between occlusion and EMG activity of the masseter muscles during clenching at maximal intercuspal position: a comparative study between prognathics and controls. $J$. Craniomandibular Pract., 20(2):99-104, 2002.

Deguchi, T.; Kumai, T. \& Garetto, L. Statistics of differential lissajous EMG for normal occlusion and class II malocclusion. Am. J. Orthod. Dentofacial Orthop., 105:42-48, 1994.
Ferrario, V.; Sforza, A.; Miani, A.; D’Addona, A. \& Barbini, E. Electromyographic activity of human masticatory muscles in normal young people. Statistical evaluation of reference values for clinical applications. J. Oral Rehabil., 20:271-80, 1993.

Grando, G.; Young, A.; Vedovello, M. \& Ramirez, O. Prevalence of malocclusion in a young Brazilian population. Int. J. Orthod. Milwaukee., 19(2):13-6, 2008.

Haraldson, T.; Carlson, G.; Dahlstrom L \& Jansson T. Relationship between myoelectric activity in masticatory muscles and bite force. Scand. J. Dent. Res., 93:539545,1985 . 
Hebling, S.; Cortellazzi, K.; Tagliaferro, E.; Hebling, E.; Ambrosano, G.; Meneghim, M. \& Pereira, A. Relationship between malocclusion and behavioural demographic and socioeconomic variables: acrosssectional study of 5 years-olds. Int. J. Orthod. Milwaukee, 19(2):13-6, 2008.

Miralles, R.; Hevia, R.; Contreras, L.; Carvajal, R.; Bull, R. \& Manns, A. Patterns of electromyographic activity in subjects with different skeletal facial types. Angle Orthod., 61(4):277-84, 1991.

Moreno, I.; Sanchez, T.; Ardizone, I; Aneiros, F. \& Celemin, A. Electromyographic comparisons between clenching, swallowing and chewing in jaw muscles with varying occlusal parameters. Med. Oral Patol. Oral Cir. Bucal, 13(3):207-13, 2008.

Moyers, M. Manual de Ortodoncia. 4ª edición. Buenos Aires, Editorial Panamericana, 1992.

Ogalde, A. \& Cardenas, H. Frecuencia de pseudoprognatismo y actividad del músculo masetero en pacientes figurados. Revista Médica de Chile, 128:1005-10, 2000.

Moreno, I.; Sanchez, T.; Ardizone, I; Aneiros, F. \& Celemin, A. Electromyographic comparisons between clenching, swallowing and chewing in jaw muscles with varying occlusal parameters. Med. Oral Patol. Oral Cir. Bucal, 13(3):207-13, 2008.

Moyers, M. Manual de Ortodoncia. $4^{\mathrm{a}}$ edición. Buenos Aires, Editorial Panamericana, 1992.

Ogalde, A. \& Cardenas, H. Frecuencia de pseudoprognatismo y actividad del músculo masetero en pacientes figurados. Revista Médica de Chile, 128:1005-10, 2000.

Pancherz, H. Activity of the temporal and masseter muscles in class II division 1 malocclusions. Am. J. Orthod. Dentofacial Orthop., 77(6):679-88, 1980a.

Pancherz, H. Temporal and masseter muscle activity in children and adults with normal occlusion. Acta Odontol. Scand., 38:343-8, 1980b.

Robertson, G.; Caldwell, B.; Hamill, J. \& Kamen, G. Research methods in biomechanics. Champaign, IL., Human Kinetics, 2004. pp.163-80.

Sabashi, K., Saitoh, I.; Hayasaki, H.; Iwase, Y.; Kondo, S.; Inada, E.; Takemoto, Y.; Yamada, C. \& Yamasaki, Y. A cross-sectional study of developing resting masseter activity in different angle classifications in adolescence. Cranio, 27(1):39-45, 2009.

Sidlauskas, A. \& Lopatiene, K. The prevalence of malocclusion among 7-15-years-old lithuanian Schoolchildren. Medicina (Kaunas), 45(2):147-52, 2009.

Silvestre, R. Electromiografía de superficie y fatiga muscular local. Revista de Kinesiología, 69:114 -9, 2002.

Throckmorton, G.; Finn, R. \& Bell, W. Biomechanics of differences in lower facial height. Am. J. Orthod., 77(2):410-20, 1980.

Traebert, E. \& Peres, M. Prevalence of malocclusion and their impact on quality of life of 18-year-old young male adults of Florianapólis. Oral Health Prev. Dent., 3(4):217-24, 2005.

Turkawski, S. \& Van Eijden, T. EMG power spectrum and motor unit characteristics in the masseter muscle of the rabbit. J. Dent. Res., 79(4):950-6, 2000.

Dirección para correspondencia:

Profesora María Eliana Albornoz Verdugo

Facultad Ciencias de la Salud

Universidad Autónoma de Chile -Talca

5 poniente $1670,5^{\circ}$ piso, Talca

CHILE

Email: mealbornoz@uautonoma.cl

Recibido : 01-07-2009

Aceptado: 12-08-2009 